Kern, Friederike; Schwier, Volker; Stövesand, Björn

\title{
Zum Ungleichgewicht digital vermittelten Sachunterrichts und
}

\section{sprachlich-kommunikativer Anforderungen}

Sachunterricht in der Informationsgesellschaft. Bad Heilbrunn : Verlag Julius Klinkhardt 2022, S. 114-121. (Probleme und Perspektiven des Sachunterrichts; 32)

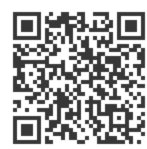

Quellenangabe/ Reference:

Kern, Friederike; Schwier, Volker; Stövesand, Björn: Zum Ungleichgewicht digital vermittelten

Sachunterrichts und sprachlich-kommunikativer Anforderungen - In: Sachunterricht in der

Informationsgesellschaft. Bad Heilbrunn : Verlag Julius Klinkhardt 2022, S. 114-121 - URN:

urn:nbn:de:0111-pedocs-242070 - DOI: 10.25656/01:24207

\section{https://nbn-resolving.org/urn:nbn:de:0111-pedocs-242070}

https://doi.org/10.25656/01:24207

in Kooperation mit / in cooperation with:

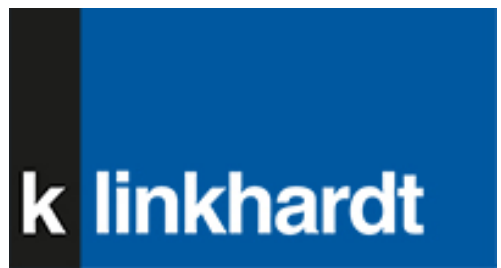

http://www.klinkhardt.de

\section{Nutzungsbedingungen}

Dieses Dokument steht unter folgender Creative Commons-Lizenz: http://creativecommons.org/licenses/by-nc-sa/4.0/deed.de - Sie dürfen das Werk bzw. den Inhalt unter folgenden Bedingungen vervielfältigen, verbreiten und öffentlich zugänglich machen sowie Abwandlungen und Bearbeitungen des Werkes bzw. Inhaltes anfertigen: Sie müssen den Namen des Autors/Rechteinhabers in der von ihm festgelegten Weise nennen. Dieses Werk bzw. der Inhalt darf nicht für kommerzielle Zwecke verwendet werden. Die neu entstandenen Werke bzw. Inhalte dürfen nur unter Verwendung von Lizenzbedingungen weitergegeben werden, die mit denen dieses Lizenzvertrages identisch oder vergleichbar sind.

Mit der Verwendung dieses Dokuments erkennen Sie die Nutzungsbedingungen an.

\section{Terms of use}

This document is published under following Creative Commons-License: http://creativecommons.org/licenses/by-nc-sa/4.0/deed.en - You may copy, distribute and transmit, adapt or exhibit the work in the public and alter, transform or change this work as long as you attribute the work in the manner specified by the author or licensor. You are not allowed to make commercial use of the work. If you alter, transform, or change this work in any way, you may distribute the resulting work only under this or a comparable license.

By using this particular document, you accept the above-stated conditions of use.

\section{Kontakt / Contact:}

\section{peDOCS}

DIPF | Leibniz-Institut für Bildungsforschung und Bildungsinformation Informationszentrum (IZ) Bildung

E-Mail: pedocs@dipf.de

Internet: www.pedocs.de

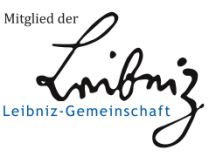




\section{Probleme und Perspektiven}

des Sachunterrichts



Andrea Becher / Eva Blumberg / Thomas Goll / Kerstin Michalik / Claudia Tenberge (Hrsg.)

Sachunterricht in der Informationsgesellschaft 
Probleme und Perspektiven

des Sachunterrichts

Band 32 


\section{Andrea Becher Eva Blumberg Thomas Goll \\ Kerstin Michalik Claudia Tenberge (Hrsg.)}

\section{Sachunterricht in der Informationsgesellschaft}


Schriftenreihe der

Gesellschaft für Didaktik des Sachunterrichts e.V.

Die Gesellschaft für Didaktik des Sachunterrichts (GDSU) e.V. ist ein Zusammenschluss von Lehrenden aus Hochschule, Lehrerfortbildung, Lehrerweiterbildung und Schule. Ihre Aufgabe ist die Förderung der Didaktik des Sachunterrichts als wissenschaftliche Disziplin in Forschung und Lehre sowie die Vertretung der Belange des Schulfaches Sachunterricht. www.gdsu.de

Bibliografische Information der Deutschen Nationalbibliothek Die Deutsche Nationalbibliothek verzeichnet diese Publikation in der Deutschen Nationalbibliografie; detaillierte bibliografische Daten sind im Internet abrufbar über http://dnb.d-nb.de.

2022.d. () by Julius Klinkhardt.

Druck und Bindung: Friedrich Pustet, Regensburg.

Printed in Germany 2022.

Gedruckt auf chlorfrei gebleichtem alterungsbeständigem Papier. 


\section{Inhaltsverzeichnis}

Andrea Becher, Eva Blumberg, Thomas Goll,

Kerstin Michalik und Claudia Tenberge

Editorial

Sachunterricht in der Informationsgesellschaft:

Sachunterrichtsdidaktische Rekonstruktion -

Medienbildung - informatische Bildung

Friedrich Gervé

Sachunterricht in der Informationsgesellschaft 17

Henrike Friedrichs-Liesenkötter

Die Ungleichheit und inklusive Medienbildung anhand

einer Betrachtung des Konnexes von Medien - Bildung - Flucht

Carsten Schulte

Digitale Technologien und informatische Bildung im Sachunterricht der Grundschule.

Sachunterricht in der Informationsgesellschaft:

Orte - Medien - Technologien

Michael Haider, Markus Peschel, Thomas Irion, Inga Gryl,

Daniela Schmeinck und Martin Brämer

Die Veränderung der Lebenswelt der Kinder und ihre Folgen

für Sachunterricht, Lehrkräftebildung und

sachunterrichtsdidaktische Forschung

Swaantje Brill und Alexandra Flügel

Digital unterwegs - außerschulische Lernorte

im Kontext digitaler Praktiken 
Svantje Schumann und Corinne Ruesch Schweizer

App-basierte Erfahrung und Reflexion als Unterstützung

der Professionalisierung von Lehrpersonen im Bereich BNE

Pascal Kihm und Markus Peschel

Gute Aufgaben 2.0 - Aufgaben und Aufgabenkulturen

im Rahmen der Digitalisierung

\section{Sachunterricht in der Informationsgesellschaft:}

Projekte im Kontext universitärer Lehrerbildung

Julia Peuke, Detlef Pech und Jara Urban

Zeitzeug*innengespräche und historisches Lernen

im Sachunterricht - ein Projektseminar.

Andreas Schmitt

Einfluss von Vorerfahrungen auf die

Fähigkeitsselbstkonzepte von Sachunterrichtsstudierenden

zu Kompetenzen in der digitalen Welt.

Friederike Kern, Volker Schwier und Björn Stövesand

Zum Ungleichgewicht digital vermittelten Sachunterrichts

und sprachlich-kommunikativer Anforderungen

Martin Brämer, Daniel Rehfeldt und Hilde Köster

Computational Thinking bei Sachunterrichtsstudierenden

im Lehr-Lern-Labor - Eine Rasch-Analyse

\section{Sachunterricht in der Informationsgesellschaft: \\ Perspektivenbezogene Zugangsweisen}

\section{Thomas Goll}

Political Literacy von Kindern -

Befunde, Implikationen, Herausforderungen

Melanie Haltenberger, Florian Böschl und Katharina Asen-Molz

Das Modell der Didaktischen Rekonstruktion als Kriterienraster

für studentische Erklärvideos nutzen - Ergebnisse aus einem standortübergreifenden Seminar zur geographischen Perspektive 
Josua Dubach, Natalie Schelleis, Katrin Bölsterli, Trix Cacchione,

Corinna S. Martarelli, Matthias Probst und Sebastian Tempelmann

Unzugängliche Welten für das erfahrungsbasierte Lernen erschließen:

Immersive Virtuelle Realität im naturwissenschaftlichen Sachunterricht........147

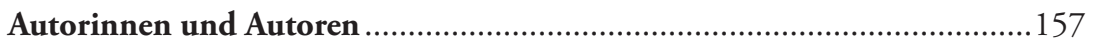


Friederike Kern, Volker Schwier und Björn Stövesand

\section{Zum Ungleichgewicht digital vermittelten Sachunterrichts und sprachlich-kommunikativer Anforderungen}

In the light of theoretical assumptions concerning the role of communication for the co-construction of knowledge, we identify a conflict between claims of science and social studies at primary level, and digitally mediated teaching: There seems to be a significant imbalance between written and oral formats in digital teaching situations. However, not only is orality assumed important for the acquisition of linguistic and content-related knowledge, negotiating content and positions is an integral part of science and social studies. Drawing on statements by primary school teachers, we argue that the observed preponderance of written tasks formats without any chance for direct exchange might furthermore reinforce processes of social inequality.

\section{Einleitung}

Der Mündlichkeit und dem argumentativen Ver- und Aushandeln von Inhalten und Positionen als konstitutives Element kommen im Sachunterricht eine bedeutende Rolle zu (Rank, Wildemann \& Hartinger 2016). Schüler*innen eignen sich Sachverhalte oft zunächst in medialer und konzeptioneller Mündlichkeit an, bevor sie sie unter Anleitung und mit Unterstützung in schriftnähere Formen übertragen. In der Zeit des pandemiebedingten Distanzunterrichts und digitaler Lehr-Lernformate erfolgte die Kommunikation vermehrt über schriftliche Aufgabenformen. Der damit einhergehende Verlust an direkter mündlicher Interaktion und Interaktionsformen riskiert die Aneignung wichtiger Sprach- und Sachkompetenzen medial zu vereinseitigen, damit zu erschweren und bestehende soziale Ungleichheiten weiter zu verstärken. Diese Problematik greift der Beitrag auf, indem er theoretische Überlegungen zur Rolle von kommunikativen Interaktionen für die gemeinsame Konstitution von Wissen mit Äußerungen von Grundschullehrkräften kontrastiert. Dadurch wird ein Konflikt zwischen sachunterrichtsdidaktischen Ansprüchen und digital vermittelter Unterrichtswirklichkeit sichtbar. Im nächsten Schritt richten wir den Blick auf mögliche Konsequenzen, die sich 
aus diesem Dilemma für die (Aus-)Bildung von Studierenden und hochschuldidaktische Konzeptualisierungen im Bereich sachunterrichtsbezogener Lehrer*innenbildung ergeben. Ausgehend von eigenen Arbeiten (Schüssler, Schöning, Schwier, Schicht, Gold \& Weyland 2017; Kern \& Stövesand 2019) diskutieren wir abschließend im Rahmen des Forschenden Lernens qualitativ-rekonstruktive Zugänge in ethnografischer Tradition, durch deren wissenschaftliche Wahrnehmungs- und Erkenntnispraktiken ein Sensibilisierungsprozess angeregt werden kann, der Studierende befähigt, das genannte Spannungsfeld sowohl reflexiv zu erfassen als auch produktiv zu bearbeiten. Zitate aus einem Gespräch mit einer Lehrkraft aus dem Corona-Sommer 2021 illustrieren die beschriebenen Problematiken bezüglich der Rolle von kommunikativen Interaktionen für den Wissenserwerb allgemein und für die Vermittlung sachunterrichtsdidaktischer Ziele im Besonderen. ${ }^{1}$

\section{Der Sachunterricht als sprachlich-soziale Interaktion}

Was schon für den Unterricht (in der Primarstufe) generell gilt, erfährt im Kontext des Sachunterrichts besondere Relevanz:

„Gemeinsames Lernen hat Anregungspotential durch die kognitive Vielfalt, wenn Wissen in sozialer Ko-Konstruktion - kognitiv aktiviert - gemeinsam ausgehandelt wird und/oder Modelllernprozesse stattfinden." (Munser-Kiefer 2019, 105)

Doch im Zuge einer sich ausweitenden digital vermittelten Unterrichtsrealität kommt es zu einer stärkeren Individualisierung von Lernprozessen. Sachunterrichtsdidaktische Ansprüche werden so teils unterlaufen:

„Im Sachunterricht erfahren die Kinder, dass nicht mehr das, was man für sich alleine weiß und für richtig hält, als Wissen gilt, sondern zunehmend das, was auch andere verstehen, einsehen und nutzen können. In der Interaktion mit anderen, denen man etwas erklärt oder plausibel machen muss, mit deren Einwänden man umgeht und deren Vorschläge man abwägt, annimmt, verwirft, wird Wissen, auf das man vertraut, einer Bewährung unterzogen." (Kahlert 2016, 138)

1 In diesem Sinne entsprechen wir in unserem Beitrag den ethnografischen Gedanken, die weder bestimmte Arten von Datenkorpora noch typische Verfahrensschritte vorschreiben, schon gar nicht in Richtung vermeintlich umfänglicher Quantifizierung oder statistischer Repräsentativität. Stattdessen folgen wir dem Ziel, durch verschiedene Datentypen die Komplexität eines Phänomens sichtbar zu machen oder sogar zu erhöhen (Breidenstein, Hirschauer, Kalthoff \& Nieswand 2020, 38 f.). So dient das Gespräch mit der Lehrkraft als Ausgangs- und Fluchtpunkt unserer sonst eher theoretischen Diskussion um die Rolle von Kommunikation und Interaktion im Sachunterricht. 
Besonders das gemeinsame Lernen eröffnet Interaktionen, in denen sachunterrichtliches Wissen von den Lernenden ko-konstruiert werden kann, so wie es auch eine von uns befragte Lehrkraft einfordert (siehe Zitat am Ende dieses Abschnitts). Sachunterricht zielt also darauf ab, einerseits entsprechende (individuelle) Erfahrungen zu initiieren und andererseits Möglichkeiten zum Austausch von Erfahrungen in sozialen Interaktionen zu eröffnen. Dieser fachdidaktische Anspruch ist insofern folgenreich, als damit

„Wissen [...] nicht mehr nur bezogen auf eigene Lebensweltprobleme und -interpretationen gültig sein [soll]. Zugleich wird der Gebrauch der Symbole, mit denen Wissen kommuniziert wird (spezielle Wortbedeutungen, Fachausdrücke, mathematische Symbole) spezifischer. Man kann sein Wissen nicht mehr, irgendwie' ausdrücken, sondern muss sich an Regeln halten, die allgemein verstanden und akzeptiert werden" (a.a.O.).

Grundsätzlich stellt auch Tänzer $(2015,449)$ fest:

„Sacherschließungsprozesse sind Verstehensprozesse. Ihr Gelingen ist entsprechend danach zu beurteilen, ob aus der Begegnung mit einer Sache eine fruchtbare, aktive Auseinandersetzung wird, innerhalb derer den Kindern ihre individuellen Wahrnehmungs-, Deutungs- und Interpretationsmuster bewusst und zum Ausgangspunkt des Auf- und Ausbaus stabiler, intersubjektiv gültiger, allgemeiner Sinn- und Sachzusammenhänge (nach Maßgabe fachwissenschaftlicher Deutungen und Erklärungen) werden. Es wird sichtbar an der Fähigkeit, ihr Verständnis auch diskursiv vertreten zu können.“

Didaktische Erwartungen zum Modus sachunterrichtlicher Lernprozesse stehen so in einem auffälligen Kontrast zu jener sachunterrichtlichen Gegenwart, wie sie von der von uns befragten Lehrkraft wahrgenommen wird:

„(...) bisschen was erklären lassen oder so kann man ja. Aber Sachunterricht ist ja ganz oft auch Experimente machen und erklären warum ist das so (...) oder vermuten (...) ich vermute das dann überlegen wir was passiert (...) dann machen wir das (...) und dann reflektieren wir was ist denn jetzt wirklich passiert und warum ist das so. Das fällt ja alles weg. Das ist ja jetzt nur Arbeitsblätter machen (...) ähm (...) und das wars." (Zitat 1)

Das Unbehagen der Lehrkraft darüber - angesichts der coronabedingten Distanzund Wechsel-Lehr-Lernformate - den genuinen sachunterrichtsdidaktischen Erwartungen wohl kaum entsprechen zu können, ist gut begründet, denn

„Gespräch und kommunikativer Austausch unterschiedlicher Form sind [...] konstitutiver methodischer Bestandteil jeder Sacherschließung" (Tänzer 2015, 449).

Wenn nun im Zuge der digital vermittelten Unterrichtspraktiken besonders die schriftfixierten Aufgaben- und Bearbeitungsformate ausgeweitet werden, während die Gelegenheiten mündlicher Teilhabe für die Schüler*innen reduziert sind, besteht jedoch das Risiko, die Aneignung wichtiger Sprach- und Sachkompetenzen 
medial zu vereinseitigen. Mit Blick auf bildungssprachliche Praktiken entfallen so bspw. Möglichkeiten des Scaffoldings (Gibbons 2006) und damit einer situierten Transformation konzeptionell mündlicher Sprachformen in bildungssprachliche. Das gemeinsame Lernen als möglicher Ort für solche Transformationen droht in digital vermittelten Lehr-Lernformaten zunehmend marginal zu werden.

\section{Die Sprache als Werkzeug des Denkens}

Anknüpfend an Zielsetzungen des Sachunterrichts und der Rolle des sprachlich-kommunikativen Austauschs für Sacherschließungsprozesse werden nun zwei der Funktionen von Sprache und Kommunikation in Lehr-Lern-Prozessen betrachtet. Erstens wird der Sprache eine wesentliche Bedeutung bei der Vermittlung und Aneignung sach- und fachbezogenen Wissens zugeschrieben; zweitens wird Wissen in sprachlich-kommunikativen Interaktionen von den Teilnehmenden gemeinsam konstruiert, wie die ethnomethodologisch inspirierte Interaktionsforschung empirisch belegt (Bergmann \& Quasthoff 2010). Insbesondere im Zusammenhang mit der Diskussion um bildungssprachliche Praktiken wird die epistemische, d.h. auf Erkenntnis bezogene Funktion von Sprache im Unterricht hervorgehoben (Morek \& Heller 2012). Das Erlernen fachlicher Termini führe einerseits dazu, dass die entsprechenden Konzepte ebenfalls angeeignet werden: „learning science is the same as learning the language of science" (Halliday 1993, 77). Andererseits zeigt sich gerade bei der Verwendung von Fachbegriffen, ob bzw. inwieweit die jeweiligen Konzepte überhaupt korrekt erschlossen wurden (Ortner 2009, 2231). Für eine korrekte Begriffsbildung, die mit Wissenszugewinn einhergeht, sind also bildungssprachliche Praktiken - d.h. in interaktive Zusammenhänge eingebettete und damit situierte und kontextbezogene sprachliche Handlungen - besonders relevant (Kern, Schwier \& Stövesand 2021). Dies führt uns zu der zweiten Funktion von Sprache und Kommunikation in Lehr-Lern-Prozessen. Zwar ist Wissen im allgemeinen Verständnis zunächst ein kognitives und personenbezogenes Konzept (z.B. Kahlert 2016, 138), jedoch spielen sprachlich-kommunikative Prozesse bei seiner Herstellung und Vermittlung eine wichtige Rolle. Dass und wie Wissen in Interaktionen gemeinsam hergestellt wird, zeigen z.B. Studien aus dem rekonstruktiven Paradigma. Gerade die mündlich-diskursive und multimodale Auseinandersetzung mit fachlichen Inhalten hat offenbar ein hohes Potenzial für die Aneignung fachlichen Wissens (z.B. Dalgren 2017). Aus dieser Perspektive ist die Bedeutsamkeit kommunikativen Austauschs zur Aneignung von Wissen nicht zu unterschätzen. Nun böte der digitale Fernunterricht zwar die Möglichkeit, kommunikativen Austausch mit Hilfe technischer Tools zu organisieren, dies wird aber, wie die bereits angeführte Aussage der Lehrkraft 
zeigt, offenbar kaum genutzt. „Das ist ja jetzt nur Arbeitsblätter machen (...) ähm (...) und das wars. " (Zitat 2) Aus diesem Zitat sowie aus Gesprächen mit weiteren Lehrkräften lässt sich als vorläufige Hypothese ableiten, dass Formen kommunikativen Austauschs im pandemiebedingt digital vermittelten Sachunterricht wohl eine eher untergeordnete Rolle spielen (wenn sie überhaupt erfolgen). Ebenso wenig werden offenbar digitale Tools für einen informellen Austausch genutzt, wie z.B. Messenger-Dienste, die auch auditive (Sprach-)Nachrichten ermöglichen. So werden gerade die jüngeren Kinder systematisch aus der Kommunikation untereinander sowie mit Lehrkräften ausgeschlossen; diese konzentriert sich stattdessen auf den Austausch mit den Eltern. Auf die Frage, wieviel Interaktion im Sachunterricht aktuell schriftlich ablaufe, antwortet die befragte Lehrkraft:

„Mit den Kindern kaum. Mit den Eltern viel, weil gut die Einser können noch nicht schreiben ähm und die anderen halt über diese Chatfunktion. Aber ähm (...) vielleicht (...) zwan_zehn_zwanzig prozent? Ganz wenig." (Zitat 3)

Die Auslagerung der mündlichen (Wissens-)Kommunikation in die Familie kann wiederum der Verschärfung sozialer Ungleichheit Vorschub leisten, da Familienkommunikation eine wesentliche Rolle beim Erwerb schulrelevanter diskursiver Fähigkeiten spielt (z.B. Kern \& Quasthoff 2007). Ausgehend von diesen Überlegungen erörtern wir nun, wie dem durch eine Sensibilisierung für fachbezogene Sprachpraktiken bereits bei Studierenden des Lehramts im Rahmen Forschenden Lernens entgegenwirkt werden kann.

\section{Ethnografisch inspiriertes Forschen über Sachunterricht}

Die vorherigen Ausführungen haben gezeigt, dass der Sachunterricht in besonderem Maße eine reflexive Auseinandersetzung mit den unterschiedlichen epistemischen Konfigurationen von Sprache notwendig macht. Bildungssprachliche Praktiken, wie z.B. Experimentierhinweise, Bildkartenbeschreibungen, Lernspielanleitungen, Forscher*innentagebücher u.Ä. konzentrieren sich oft ebenfalls auf die Vermittlung von Fachtermini, sind dabei jedoch konstitutiver Teil der Fachlichkeit im Sachunterricht. Dennoch muss sich die Sachunterrichtsdidaktik auf besondere Weise mit Sprache und Sprachgebrauch auseinandersetzen, wie das Zitat 1 der Lehrkraft eindrücklich zeigt. So wird von ihr vor allem die Relevanz der Operatoren „vermuten “, „überlegen“ und „reflektieren“ angesprochen, welche sich mit den schultypischen Praktiken des „Erklärens“, „Begründens“ und „Argumentierens" identifizieren lassen (auch GDSU 2013, 12 ff.) - alles Praktiken, die im (Sach-) Unterricht zunächst vornehmlich mündlich auftreten. Beim Erwerb und Üben solcher mündlicher ,bildungssprachlicher Praktiken' geht es jedoch nicht ausschließlich um das Einüben von probater Lexik und komplexer Syntax, 
sondern vor allem um einen diskursiv angemessenen, situierten und damit kontextualisierten Gebrauch der entsprechenden Praktiken (Morek \& Heller 2012). Der zunehmende Einsatz digitaler Tools oder gar der vollständige Wechsel in einen medial vermittelten Distanzunterricht während der Corona-Pandemie macht, neben der kritischen Auseinandersetzung mit interaktionsrelevanten Konsequenzen dieser Tools für den Unterricht, eine Beschäftigung mit den skizzierten fachlich-sprachlichen Implikationen notwendig. Unser Vorschlag schließt hier an eine Verbindung aus Forschendem Lernen (Fichten 2010) und der Kasuistik an (Schmidt \& Wittek 2020) an. Durch einen fallorientierten und vornehmlich rekonstruktiven Zugang sollen angehende Lehrkräfte so an prinzipiell qualitativ-wissenschaftlichen Praktiken partizipieren und darüber die Fähigkeit zur kritisch-reflexiven Distanz entwickeln. Dabei folgen wir einem hochschuldidaktischen Konzept, das im Teilprojekt 3 („Fachdidaktische Professionalisierung unter Berücksichtigung sozialer Ungleichheit und Inklusion") des BiProfessional-Projekts ${ }^{2}$ entwickelt wurde und in der Fachdidaktik Deutsch bereits im Umfeld der studentischen Praxisaufenthalte eingesetzt wird (Kern \& Stövesand 2019). Darin führen Studierende eigenständig ethnografisch inspirierte Beobachtungen durch, um so einen Verfremdungseffekt zu erzielen zugunsten eines Blicks für die fachspezifische Mikrokultur, wie sie im interaktiven Vollzug des Unterrichts hergestellt wird. Mit Blick auf den Sachunterricht kann ein ethnografisch inspiriertes Erforschen seitens der Studierenden den Blick dafür weiten, wie sich die Rolle der Sprache in der Unterrichtspraxis durch die Zunahme digitaler Hilfsmittel wandelt und welche potenziellen Konsequenzen dies für den Wissenserwerb seitens der Schüler*innen hat. Als forschend Teilnehmende an der Unterrichtssituation haben angehende Lehrkräfte so die Möglichkeit, den Entschleunigungs- und Verfremdungseffekt von wissenschaftlichen Beobachtungsformaten zu nutzen und die dabei entstehenden Daten (Protokolle, Transkripte, etc.) analytisch zu durchdringen. So können bspw. auf Basis von detaillierten Beobachtungsprotokollen, die auf Relationierung von mündlichem Unterrichtsgespräch und schriftlichen Aufgabenstellungen und Gegenstandspräsentationen im Digitalen fokussieren, fachliche Spannungsfelder identifiziert werden. Dabei kann es z.B. gelingen, grundlegende Differenzen oder Widersprüche im mündlichen wie (konzeptionell) schriftsprachlichen Vollzug von Unterrichtsexperimenten zu reflektieren. Als Teilnehmende an der Unterrichtssituation wird so eine Reflexionsebene eingezogen, die den sachunterrichtlichen Sprachgebrauch zu einem „cognitive artifact” (Goodwin 1994, 615) werden lässt: Sprache wird als Unterrichtsmedium und sachunterrichtlicher Lerngegenstand erfahr- und analysierbar, auch in Bereichen, in denen digitale Hilfsmittel ggf. die schriftsprachliche Dimension überbetonen.

2 Der Beitrag ist in dem Projekt BiProfessional entstanden. BiProfessional wird im Rahmen der gemeinsamen Qualitätsoffensive Lehrerbildung von Bund und Ländern aus Mitteln des Bundesministeriums für Bildung und Forschung gefördert (Förderkennzeichen 01JA1908). 
Damit einher geht das Potenzial, sprachlich bedingte Ungleichheitsfaktoren in diesen neuen Unterrichtsmodalitäten zu entdecken und in ihrer jeweils situierten Relevanz zu reflektieren. So wird eine Dimension von Digitalitätskompetenz adressiert, die über das angemessene Verwenden von Tools deutlich hinausgeht: Es wird vielmehr ein analytischer Blick angeeignet, der die Herstellung einer sachunterrichtlich-sprachlichen Unterrichtsrealität im Kontext der Verwendung digitaler Hilfsmittel fokussiert und damit deren prozessualen Charakter gegenüber einer vermeintlichen Objekthaftigkeit prominent setzt. Die Kontrastierung eines sachunterrichtsdidaktisch-sprachlichen Anforderungsprofils mit Erfahrungen von (pandemiebedingt) digital vermittelter Sachunterrichtsrealität einzelner Lehrkräfte unterstreicht grundlegende Problemlagen kommunikativer Interaktion im Sachunterricht. Konstruktiv gewendet - so unser Vorschlag - können diese gleichwohl als Reflexionsanlässe genutzt werden, um Studierende zu unterstützen, den (fach- und bildungs-)sprachlichen Implikationen fachdidaktischer Entscheidungen gewahr zu werden. Obgleich herausfordernd, entstehen durch dafür notwendige Distanzierungen und Verfremdungen essenzielle professionalitätsbezogene Lerngelegenheiten.

\section{Literatur}

Breidenstein, G., Hirschauer, S., Kalthoff, H. \& Nieswand, B. (2020): Ethnografie. Die Praxis der Feldforschung. 3., überarb. Aufl., München.

Dalgren, S. (2017): Questions and answers, a seesaw and embodied action: How a preschool teacher and children accomplish educational practice. In: Bateman, A. \& Church, A. (Hrsg.): Children's knowledge-in-interaction. Studies in Conversation Analysis. Singapore, S. 37-56.

Fichten, W. (2010): Forschendes Lernen in der Lehrerbildung. In: Eberhardt, U. (Hrsg): Neue Impulse in der Hochschuldidaktik. Wiesbaden, S. 127-182.

GDSU (Gesellschaft für Didaktik des Sachunterrichts) (Hrsg.) (2013): Perspektivrahmen Sachunterricht. Vollst. überarb. und erw. Ausgabe. Bad Heilbrunn.

Gibbons, P. (2006): Unterrichtsgespräche und das Erlernen neuer Register in der Zweitsprache. In: Mecheril, P. \& Quehl, Th. (Hrsg.): Die Macht der Sprachen. Englische Perspektiven auf die mehrsprachige Schule. Münster u. a., S. 269-290.

Goodwin, C. (1994): Professional Vision. In: American Anthropologist, 96, 3, 606-633.

Halliday, M. A. K. (1993): Some Grammatical Problems in Scientific English. In: Halliday, M. A. K. \& Martin, J. R.: Writing Science: Literacy and Discursive Power. London, S. 69-85.

Kahlert, J. (2016): Der Sachunterricht und seine Didaktik. 4. akt. Auflage. Bad Heilbrunn.

Kern, F. (2020): Das Potenzial ethnographischer Fallarbeit für die Professionalitätsentwicklung angehender Lehrkräfte. In: Kotthoff, H. \& Heller, V. (Hrsg.): Ethnografien und Interaktionsanalysen im schulischen Feld. Tübingen, S. 221-246.

Kern, F. \& Quasthoff, U. (2007): „Familiale Interaktionsmuster und kindliche Diskursfähigkeit. Mögliche Auswirkungen interaktiver Stile auf diskursive Praktiken und Kompetenzen bei Schulkindern.“ In: Hausendorf, H. (Hrsg.): Gespräch als Prozess, Tübingen, S. 277-306.

Kern, F. \& Stövesand, B. (2019): Forschendes Lernen in der sprachdidaktischen Lehramtsausbildung. In: PFLB PraxisForschungLehrer*innenbildung, 1(2), 119-123. 
Kern, F., Schwier, V. \& Stövesand, B. (2021): Sprachsensible Professionalitätsentwicklung von Sachunterrichts-Studierenden. In: Franz, U., Giest., H., Haltenberger, M., Hartinger, A., Kantreiter, J. \& Michalik, K. (Hrsg.): Sache und Sprache. Bad Heilbrunn, S. 179-197.

Morek, M. \& Heller, V. (2012): Bildungssprache - Kommunikative, epistemische, soziale und interaktive Aspekte ihres Gebrauchs. Zeitschrift für angewandte Linguistik, 57, 1, 67-101.

Munser-Kiefer, M. (2019): Unterrichten in der Primarstufe. In: Kiel, E., Herzig, B., Maier, U. \& Sandfuchs, U. (Hrsg.): Handbuch Unterrichten an allgemeinbildenden Schulen. Bad Heilbrunn, S. 101-112.

Ortner, H. (2009): Rhetorisch-stilistische Eigenschaften der Bildungssprache. In: Fix, U., Gardt, A. \& Knape, J. (Hrsg.): Rhetorik und Stilistik/Rhetoric and Stylistics. Band 2. Berlin, New York, S. 2227-2240.

Rank, A., Wildemann, A. \& Hartinger, A. (2016): Sachunterricht - der geeignete Ort zur Förderung von Bildungssprache. In: www.widerstreit-sachunterricht.de, Nr. 22, Oktober 2016, https://www. widerstreit-sachunterricht.de/ebeneI/superworte/foerder/rank_ua.pdf [24.09.2021].

Schmidt, R. \& Wittek, D. (2020): Rekonstruktive Kasuistik - ein unerreichbares Ideal universitärer Lehre. Empirische Hinweise zum Widerstreit von Programmatik und Praxis. In: Wittek, D., Rabe, T. \& Ritter, M. (Hrsg.): Kasuistik in Forschung und Lehre. Erziehungswissenschaftliche und fachdidaktische Ordnungsversuche. Bad Heilbrunn, S. 261-280.

Schüssler, R., Schöning, A., Schwier, V., Schicht, S., Gold, J. \& Weyland, U. (Hrsg.) (2017): Forschendes Lernen im Praxissemester. Zugänge, Konzepte, Erfahrungen. Bad Heilbrunn.

Tänzer, S. (2015): Die Sachen erschließen. In: Kahlert, J., Fölling-Albers, M., Götz, M., Hartinger, A., Miller, S. \& Wittkowske, S. (Hrsg.): Handbuch Didaktik des Sachunterrichts. 2. überarb. und akt. Aufl. Bad Heilbrunn, S. 448-457. 